\title{
DETECTING LOW-INTENSITY MAGNETIC FIELDS WITH A MAGNETOSTRICTIVE FIBER OPTIC SENSOR
}

\author{
Laura L. Picon, Capt, USAF, Victor M. Bright, and Edward S. Kolesar*
}

\author{
Air Force Institute of Technology \\ Department of Electrical and Computer Engineering \\ Wright-Patterson AFB, OH 45433-7765 \\ *Texas Christian University \\ Department of Engineering, Campus Box 5100, South University Drive \\ Fort Worth, TX 76129-5100
}

\begin{abstract}
A magnetostrictive fiber optic Mach-Zehnder interferometric sensor that detects low-intensity magnetic fields was realized. The sensor utilizes $850 \mathrm{~nm}$ singlemode fiber optic cable and couplers. Four classes of sensing arms were fabricated by coupling the magnetostrictive materials to a length of fiber optic cable as: ribbons, cylinders, sandwiches, and sputter coated sheaths. The sensing arms were evaluated using Metglas $^{T M}$, nickel, and a combination of Metglas ${ }^{T M}$ and nickel. A Helmholtz coil was used to generate AC magnetic test and evaluation field. Optimization techniques were applied to the sensor to maximize its sensitivity, including: annealing the magnetostrictive material; enclosing the Helmholtz coil, sensing arm, and reference arm in a magnetic field shielded chamber; and applying a DC bias to the Helmholtz coil's AC current component. The measurements indicated that the lowest magnetic flux density that could be detected was $0.3 \mathrm{mG}$. This was achieved using a Metglas ${ }^{T M}$ ribbon-fiber configuration where ribbon's magnetization direction was aligned perpendicularly with respect to the applied $52 \mathrm{kHz} \mathrm{AC} \mathrm{mag-}$ netic field and a 5 volt DC bias. When this configuration was correspondingly operated with a $10 \mathrm{~Hz}$ AC magnetic field and 5 volt DC bias, an $8.9 \mathrm{mG}$ magnetic flux density was detected.
\end{abstract}

\section{INTRODUCTION}

The research and applications of fiber optic sensors continues to rapidly expand. In particular, the U.S. military is interested in applying fiber optic sensor technology to detect and quantify low-intensity magnetic fields generated by the human body. Measurement of these fields could supply valuable information concerning the state of a pilot's workload, stress, and consciousness [1]. Such a device could also be used to measure low-intensity magnetic fields generated from other sources. In general, the detection of low-intensity magnetic fields is a difficult problem because the magnitudes of the flux densities typically range from $10^{-9}$ to $10^{-8}$ Gauss $(G)[1]$.

Currently, low-intensity magnetic flux densities on the order of $10^{-10}$ to $10^{-4} \mathrm{G}$ are detectable with a Superconducting Quantum Interfezence Device (SQUID) [2]. However, the massive sive, prohibitive cost, and complex operation of these cryogenicallycooled devices limit their applicability to specialized laboratory settings. On the other hand, fiber optic sensors possess few of these limitations they are small, inexpensive, can operate at room temperature, and are relatively simple to manufacture and operate. For these reasons, the detection of low-intensity magnetic fields with a magnetostrictive fiber optic sensor is considered to be a potential alternative to the SQUID technology. 


\section{SENSOR DESIGN}

The detection of low-intensity magnetic fields with a magnetostrictive fiber optic sensor was first proposed by Yariv and Winsor in 1980 [3]. The first functional sensor that incorporated their design was fabricated by Dandridge in 1980 [4]. The current technology allows magnetic flux delısities as low as $1.3 \times 10^{-7} \mathrm{G}$ to be detected [5] (the author reported a detection sensitivity of $7 \times 10^{-10}(\mathrm{G} / \sqrt{\mathrm{Hz}}$ at $35 \mathrm{kHz})$.

Fabricating a magnetostrictive fiber optic sensor involves coupling a section of fiber optic cable to a magnetostrictive material. Magnetostrictive materials, which are also ferromagnetic materials, possess the inherent ability to change their physical dimensions when exposed to an externally applied magnetic field [6]. Thus, when the combination of a magnetostrictive material and fiber optic cable is exposed to a magnetic field, the magnetostrictive material will deform, causing the fiber optic cable to microbend. In turn, microbending induces a phase shift in the lightwave propagating in the sensing arm, and when this perturbed signal is combined with the unperturbed signal in the reference arm, the sensor's interference pattern is correspondingly modified. Changes in the interference pattern can be directly correlated with the intensity of the externally applied magnetic field.

Each magnetostrictive material has a distinct response to a magnetic field which equates to a unique perturbation in the length of the magnetostrictive material and the induced phase shift in the lightwave propagating in the fiber optic cable. This phase shift is given by the following equations [7]:

$$
\begin{aligned}
& \phi(H)=\frac{2 \pi n \xi}{\lambda} L \eta e(H) \\
& e(H)=C H^{2}
\end{aligned}
$$

where $n$ is the refractive index of the fiber core, $\xi$ is the strain-optic correction factor, $\lambda$ is the free-space wavelength of the propagating optical energy, $L$ is the length of fiber experiencing the strain, $\eta$ is the strain-transfer efficiency (ratio of the strain in the fiber to the strain in the magnetostrictive material), $e(H)$ is the magnetic field-dependent strain, $C$ is the magnetostrictive material parameter, and $H$ is the applied magnetic field.

Operation of the Mach-Zehnder interferometer can be explained as follows (Figure 1) [9]: 1) A 50/50ratio fiber optic coupler is used to partition the light from the optical source into the two fiber cable arms. 2) One arm, which is not coupled to the magnetostrictive material, is designated as the reference arm. 3) The other arm, which is coupled to the magnetostrictive material, is referred to as the sensing arm. When the Mach-Zehnder interferometer's reference arm and sensing arm are exposed to an externally applied magnetic field, the magnetostrictive material coupled to the sensing arm causes a deformity in the fiber optic cable which induces a phase shift in the lightwave propagating through the sensing arm. The recombination of the two lightwaves with the second 50/50-ratio fiber optic coupler results in a detectable interference pattern. This interference pattern can correspondingly be analyzed with instrumentation, such as a power meter or an optical spectrum analyzer, that displays the amplitude of the combined lightwave signals.

Research was conducted in an attempt to optimize the sensor to yield performance results comparable to those available from the SQUID. To optimize the sensor's performance, two magnetostrictive materials, Metglas ${ }^{T M}$ and nickel, were investigated. In addition, two types of single-mode fiber optic cable, Corning's non-polarization preserving Flexcore850TM and Fibercore LTD's polarization preserving HB800TM, were analyzed. Each sensor configuration was evaluated for its ability to detect $\mathrm{AC}$ magnetic fields which were generated by a Helmholtz coil.

\section{DESIGN OF SENSING ARMS}

Before coupling the fiber optic cable to the magnetostrictive material with epoxy (Super Glue Quick Epoxy Adhesive), the fiber optic cable's buffer and jacket were removed using the LiteStrip ${ }^{T M}$ buffer stripper. This was accomplished at the coupling locations to minimize the dampening effect during magnetostriction [10].

The next step in the design of the sensing arm involved the magnetostrictive materials. The magnetostrictive materials and fiber optic cables were fabricated in four distinct configurations: ribbons, sandwiches, cylinders, and coatings (Figure 2) [9]. The stripped fiber optic cable was then coupled to the ribbon, sandwich, or cylindrical geometry with epoxy. The coated fiber optic cable was realized by sputtering the magnetostrictive material onto the fiber.

The sensing arm configurations were investigated with Metglas ${ }^{T M}$ ribbon and a nickel rod serving as the magnetostrictive materials [9]. The Metglas ${ }^{T M}$ ribbon was $5.1 \mathrm{~cm}$ wide, 8 mil thick, and $10 \mathrm{~cm}$ in length. The nickel rod had a $2.5 \mathrm{~cm}$ diameter, a length of $30 \mathrm{~cm}$, and a purity of $99.1 \%$. 
The Metglas ${ }^{T M}$ ribbons were fabricated with five geometries (Figure 3) [9]. The sandwich geometry utilized a fiber optic cable sandwiched between two Metglas ${ }^{T M}$ ribbons, or the nickel rod and a Metglas $^{T M}$ ribbon. The first Metglas ${ }^{T M}$ sandwich consisted of a fiber optic cable placed between four $5 \mathrm{~cm}$ by $5.1 \mathrm{~cm}$ Metglas ${ }^{T M}$ ribbons with the orientation depicted in Figure 3(e). The second Metglas ${ }^{T M}$ sandwich consisted of a fiber optic cable placed between two, $10 \mathrm{~cm}$ long by $5.1 \mathrm{~cm}$ wide Metglas ${ }^{T M}$ ribbons with the orientation depicted in Figure 3(a). The combined Metglas ${ }^{T M}$ and nickel sandwich consisted of a fiber optic cable placed between two, $5 \mathrm{~cm}$ by $5.1 \mathrm{~cm}$ Metglas ${ }^{T M}$ ribbons oriented as shown in Figure 3(e), along with the nickel rod. Two combined Metglas $^{T M}$ and nickel sandwich configurations were fabricated: one with an unannealed nickel rod and the other with an annealed nickel rod. The combined nickel and Metglas ${ }^{T M}$ configuration was fabricated to take advantage of both materials' magnetostrictive properties. That is, when exposed to an externally applied magnetic field, the Metglas ${ }^{T M}$ expands while the nickel contracts [11].

The fiber optic cable for the ribbon and sandwich configurations involved a $6 \mathrm{~cm}$ length of the buffer stripped from a $10 \mathrm{~cm}$ long inner section of a $1 \mathrm{~m}$ long fiber optic cable.

The cylindrical shapes were generated by winding the Metglas ${ }^{T M}$ ribbons onto non-magnetic cylindrical forms (4.7 cm diameter and $11.5 \mathrm{~cm}$ long sections of a polyvinyl chloride (PVC) pipe) [9]. Once the magnetostrictive material cylinders were formed, the fiber optic cable was wound onto each cylinder. To facilitate the fiber maintaining its position, while also minimizing the amount of magnetostrictive dampening caused by the epoxy, a spiral track was machined into a subset of the Metglas ${ }^{T M}$ cylinders. The pitch of the spiral track was approximately eighty-five degrees. After this preparation, the fiber optic cable was wrapped along the machined spiral track in the Metglas $^{T M}$ cylinders.

The fiber optic cable coating scheme was accomplished by sputtering nickel onto an uncoated cable, as well as a Metglas ${ }^{T M}$ ribbon [9]. Sputtering nickel onto a Metglas ${ }^{T M}$ ribbon was accomplished to capitalize upon both materials' opposing magnetostrictive properties.

\section{INTERFEROMETER ASSEMBLY AND CALIBRATION}

The magnetostrictive fiber optic sensor was fabricated by assembling the basic Mach-Zehnder interferometer arrangement shown in Figure 1. One end of the reference arm was permanently spliced to one of the two leads of the fiber optic coupler, while the second end of the reference arm was temporarily spliced to one of the two leads of the second fiber optic coupler. Since the Mach-Zehnder interferometer's configuration was frequently changed, the sensing arm was attached between the couplers with temporary splice tubes.

As the Mach-Zehnder interferometer was assembled, the system components were individually characterized with respect to their optical performance. The performance characteristics included stability, sensitivity, and attenuation. The evaluation process also characterized the source/adapter losses, the coupler/fiber losses, and the splice losses.

\section{SYSTEM PERFORMANCE EVALUA- TION}

To simulate the magnetostrictive effect, a sensing arm was configured by winding a fiber optic cable around the circumference of a $5 \mathrm{~cm}$ wide $\mathrm{PZT}$ ring (Veritron, Model 24-32200-5A). Two biases, $4 \mathrm{~V}$ and $1 \mathrm{~V}$, were applied at $10 \mathrm{~Hz}$ to the $\mathrm{PZT}$ ring to alter its shape. The output lightwave signal was measured with and without the voltage applied to the sensor. When the two output lightwave signals were subtracted from each other, the effects of the path length difference and splice mismatch could be accounted for.

Altering the shape of the piezoelectric material wound with the fiber optic cable sensing arm caused a strain in the sensing arm. This strain induced a phase shift in the sensing arm's lightwave, which correspondingly changed the sensor's overall interference pattern. This fundamental characterization was accomplished with the single-mode non-polarization preserving fiber to demonstrate the proof of this detection concept [12] and to verify proper operation of the interferometer.

After removing the piezoelectric ring, each sensing arm was evaluated for its ability to detect lowintensity magnetic fields. Each sensing arm's performance was recorded before and during the application of a magnetic field. 


\section{SENSOR OPTIMIZATION}

The combination of fiber optic cable and magnetostrictive material that best manifested a magnetostrictive induced phase shift was further evaluated with respect to the following optimization techniques: enclosing in a magnetic field shielded chamber, thermal annealing, superimposing a DC magnetic field bias on an $\mathrm{AC}$ magnetic field signal, and varying the applied magnetic field's excitation frequency [9].

\section{a) Shielded Chamber}

To isolate the ambient magnetic flux density, an electromagnetic shielded chamber was constructed. A Plexiglas lid was fabricated to interface with the Helmholtz coil's Plexiglas base and completely enclose the Helmholtz coil arrangement [13]. The electromagnetic shielded chamber was fabricated by placing strips of co-netic AA foil (Magnetic Shield Corp.) with pressure sensitive tape backing onto the Helmholtz coil's Plexiglas lid and base. To determine the effectiveness of the shielded chamber, a Gaussmeter was used to measure the magnetic flux density inside and outside the enclosure.

\section{b) Thermal Annealing}

Thermally annealing the magnetostrictive material in the presence of a DC magnetic field was also investigated as an optimization technique. Unannealed magnetostrictive materials, such as metallic glass, exhibit local stress-induced anisotropy axes that are formed during the manufacturing process. That is, the magnetic moments of the domains of atoms in the magnetostrictive material tend to align themselves along the anisotropy axis, also known as the 'easy axis'. These anisotropy axes can reduce the sensor's sensitivity, depending upon the direction of the easy axis relative to the applied magnetic field [14]. To obtain a preferred direction of magnetization within the magnetostrictive material's bulk, the magnetostrictive material should be annealed with an externally applied DC magnetic field. The externally applied static magnetic field must be oriented transversely with respect to the longitudinal axis of the magnetostrictive material to take advantage of the maximum magnetostrictive benefits.

The Metglas ${ }^{T M}$ ribbons and sandwich materials were thermally annealed in a furnace by positioning them between the two poles of a $500 \mathrm{G}$ strength horseshoe magnet. After reaching $230^{\circ} \mathrm{C}$, the furnace was turned off, and the magnetostrictive materials placed between the two poles of the horseshoe magnet were cooled to room temperature.

\section{c) DC Magnetic Field}

During the application of this optimization technique, the total current supplied to the Helmholtz coil was composed of a DC and an AC current component. The DC offset, provided as an option on the function generator, was used to supply the DC current component. The function generator's DC offset ranges from 0 to 5 volts.

The magnetostrictive fiber optic sensor's response was evaluated for selected sensing arm configurations with a DC offset of $1,2,3,4$, and 5 volt.

\section{d) Variable Excitation Frequency}

The magnetostrictive fiber optic sensor's performance characteristics were evaluated at the following frequencies for selected sensing arm configurations: $10 \mathrm{~Hz}, 100 \mathrm{~Hz}, 1 \mathrm{kHz}, 52 \mathrm{kHz}$, and $100 \mathrm{kHz}$.

\section{SUMMARY OF SENSOR PERFOR- MANCE RESULTS}

The ribbon and cylindrical sensing arms provided the best low-intensity magnetic field detection responses. Annealing the magnetostrictive material in a furnace (under the influence of an intense static magnetic field) was found to improve the sensitivity of both the Metglas ${ }^{T M}$ and nickel, but it had a substantially larger effect on the nickel, which is known to have a random magnetic domain alignment. Positioning the Metglas $^{T M}$ in the test magnetic field to take advantage of the material's preferred magnetization direction provided the best sensing arm response for all nagnetic flux density levels.

Operation at higher frequencies improved the sensitivity of the magnetostrictive fiber optic sensor by taking advantage of the laser diode's reduced optical source intensity noise. Applying a DC bias to the $\mathrm{AC}$ magnetic field improved the response of the Metglas ${ }^{T M}$ sensing arm, but had little effect on the nickel sensing arm. The shielded chamber did not alter the performance characteristics of the sensing arm. Polarization preserving fiber optic cable reduced the signal fluctuations found in the cylindrical sensing arm's performance results, but did not improve upon the results achieved with the non-polarization preserving single-mode fiber optic cable. Combining two magnetostrictive materials, nickel and Metglas ${ }^{T M}$, did not improve the performance achieved using Metglas ${ }^{T M}$ alone. 
After the magnetostrictive fiber optic sensor was fabricated and calibrated, the measurements employing the various sensor arms indicated that the lowest magnetic flux density that could be detected was 0.3 $\mathrm{mG}$. This was achieved using a Metglas ${ }^{T M}$ ribbonfiber configuration where the ribbon's magnetization direction was perpendicularly aligned with the applied $52 \mathrm{kHz}$ AC magnetic field with the 5 volt DC bias (Figure 3.e). Figure 4 depicts the results of this particular sensing arm configuration for a 5 volt DC bias and a 2 volt DC bias [9]. The solid line represents the least-squares curve fit of the measured data. When operating this configuration with a $10 \mathrm{~Hz} \mathrm{AC}$ magnetic field and a 5 volt DC bias, the lowest magnetic flux density that could be detected was $8.9 \mathrm{mG}$.

\section{REFERENCES}

[1] R. Churchill, Laser Fiber Optic Sensor for $\mathrm{Hu}$ man Biomagnetic Measurements. Topic 86-278. Radford, VA: American Research Corporation of Virginia, 1986.

[2] J. Lenz, "A review of magnetic sensors," Proc. $I E E E$, vol. 78, pp. 973-989, June 1990.

[3] A. Yariv and H. Winsor, "Proposal for detection of magnetic fields through magnetostrictive perturbation of optical fibers," Opt. Lett., vol. 5, pp. 87-89, Mar. 1980.

[4] A. Dandridge, A. Tveten, G. Sigel, E. West, and T. Giallorenzi, "Optical fiber magnetic field sensors," Electron. Lett., vol. 16, pp. 408-409, May 1980.

[5] F. Bucholtz, D. Dagenais, and K. Koo, "High-frequency fiber-optic magnetometer with 70fT/ $\sqrt{\mathrm{Hz}}$ Resolution," Electron. Lett., vol. 25, pp. 1719-1720, Dec. 1989.

[6] M. Melamud, L. Swartzendruber, and L. Bennett, "Moment distribution in amorphous magnetic ribbons by Mossbauer measurements," $J$. Appl. Phys., vol. 61, pp. 3644-3646, Apr. 1987.

[7] F. Bucholtz, "Fiber optic magnetic sensors," in Fiber Optic Sensors, E. Udd, Ed. New York, Wiley, 1991, pp. 369-408.

[8] J. Livingston, "Magnetomechanical properties of amorphous metals," Physica Status Solidi, vol. 70, pp. 591- 596, May 1982.
[9] L.L. Picon, Detection of Low-Intensity Magnetic Fields with a Magnetostrictive Fiber Optic Sensor. MS Thesis, AFIT/GE/ENG/93D32. Graduate School of Engineering, Air Force Institute of Technology (AU), Wright-Patterson AFB OH, Dec. 1993.

[10] N. Nader-Rexvani, R. Claus, and A. Sarrafzadeh, "Low frequency fiber optic magnetic field sensors," SPIE, vol. 1584, pp. 405-414, 1991.

[11] B. White, Optical Fiber Magnetic Field Sensor Studies, October 83 - October 85. Report NADC-85154-30. Naval Air Development Center, 1985 .

[12] D. Jackson, "Elimination of drift in a singlemode optical fiber interferometer using a piezoelectrically stretched coiled fiber," Appl. Opt., vol. 19, pp. 2926-2929, Sept. 1980.

[13] D. Hackett, Investigation of a Magnetostrictive Fiber Optic Interferometric Sensor. MS Thesis, AFIT/GEO/ENG/89D-4. School of Engineering, Air Force Institute of Technology (AU), Wright-Patterson AFB OH, Dec. 1989.

[14] M. Mermelstein, "A magnetoelastic metallic glass low-frequency magnetometer," IEEE Trans. Magnetics, vol. 28, pp. 36-56, Jan. 1992. 

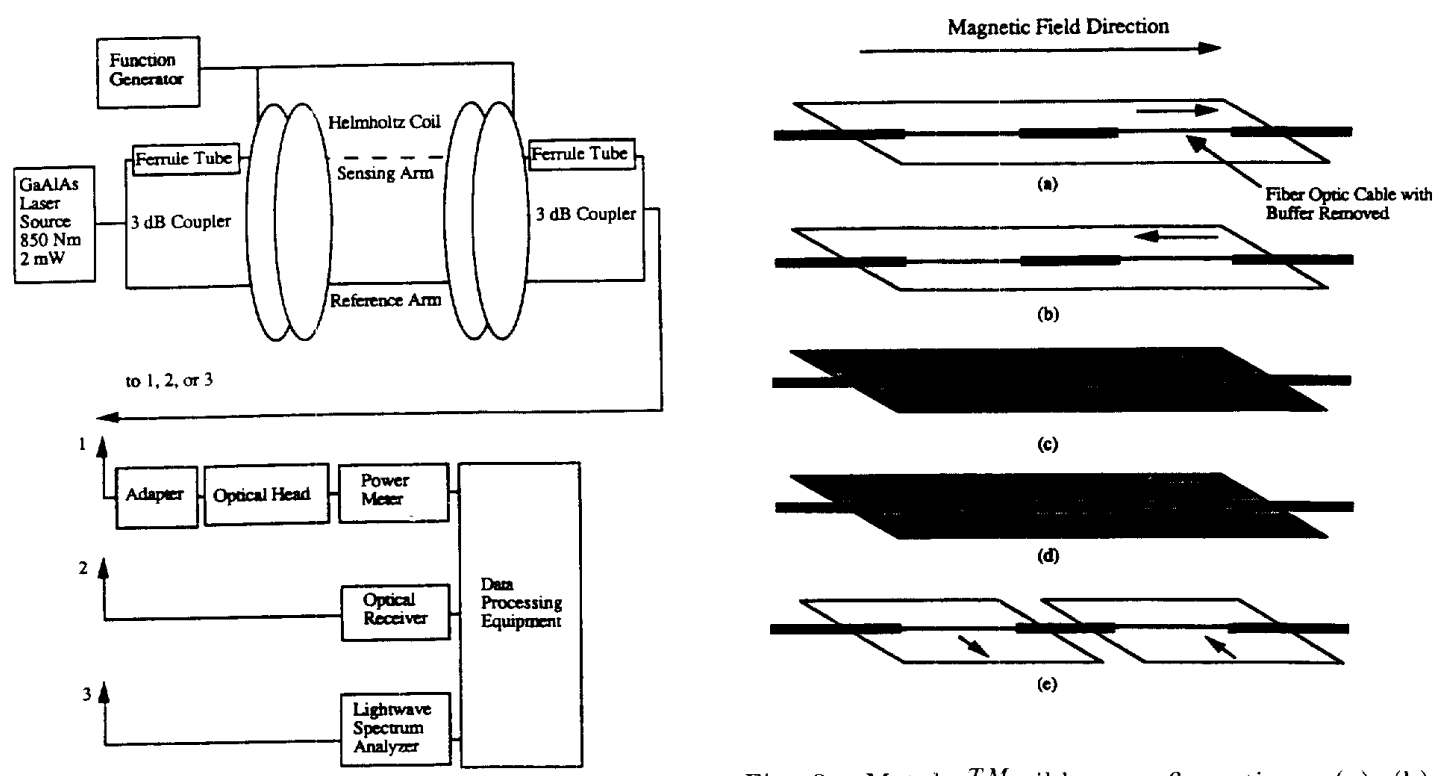

(c)

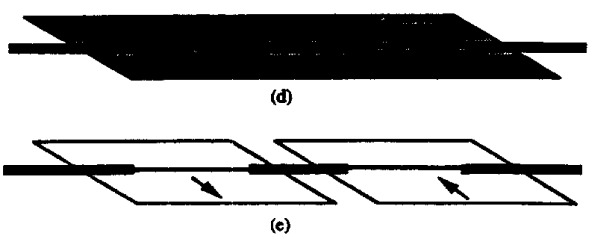

Fig. 3. Metglas ${ }^{T M}$ ribbon configurations: (a), (b), and (e) represent unannealed ribbons, (c) and (d) represent annealed ribbons. The short arrows indicate the preferred magnetic domain alignment in the magnetostrictive materials before annealing.

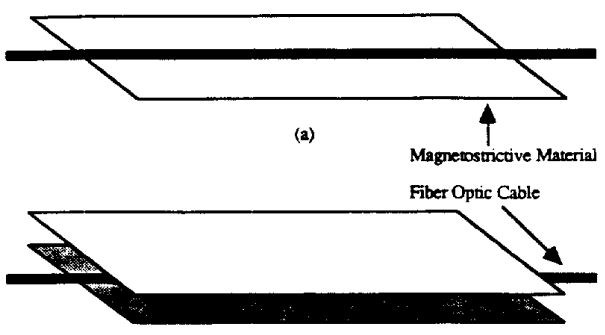

(b)
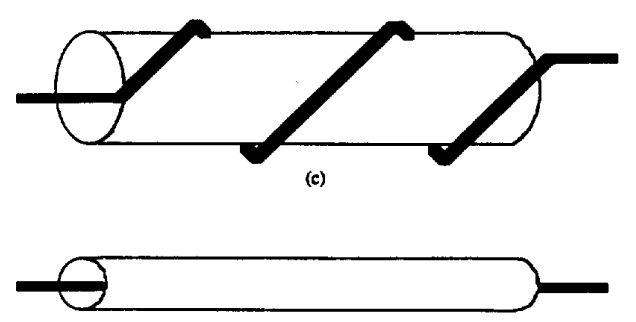

(d)

Fig. 2. Four magnetostrictive fiber optic cable geometries: (a) ribbon, (b) sandwich, (c) cylinder, and (d) external sheath coating.

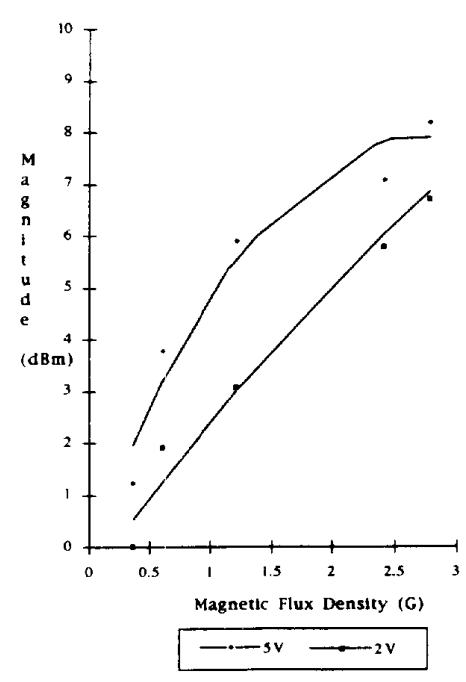

Fig. 4. Interference response for unannealed Metglas $^{T M}$ ribbon sensing cable perpendicularly aligned with respect to the $52 \mathrm{kHz}$ magnetic field (Figure 3.e). 